\title{
Validity and reliability of Kinect v2 for quantifying upper body kinematics during seated reaching
}

Germain Faity ${ }^{1},{ }^{*}$ Denis Mottet ${ }^{1}$, Jérôme Froger ${ }^{2}$

1. Euromov Digital Health in Motion, Univ Montpellier, IMT Mines Ales, Montpellier, France.

2. Euromov Digital Health in Motion, Univ Montpellier, IMT Mines Ales, CHU de Nîmes, Le Grau du Roi, France.

*Corresponding author: denis.mottet@umontpellier.fr

ORCID Germain Faity: https://orcid.org/0000-0002-6855-8938

ORCID Denis Mottet: https://orcid.org/0000-0002-7564-5394

ORCID Jérôme Froger: https://orcid.org/0000-0002-6590-3820

\section{Abstract}

Background. Kinematic analysis of the upper limbs is a good way to assess and monitor recovery in individuals with stroke, but remains little used in clinical routine due to its low feasibility. The aim of this study is to assess the validity and reliability of the Kinect v2 for the assessment of 17 kinematic variables commonly used in the analysis of upper limb reaching in stroke. Methods. 26 healthy participants performed seated hand-reaching tasks while holding a dumbbell to induce a behaviour similar to that of a person with a stroke. 3D upper limb and trunk motion were simultaneously recorded with the Kinect V2 (Microsoft, USA) and with the VICON (OxfordMetrics, UK), the latter being the reference system. For each kinematic outcome, the validity of the Kinect was assessed with ICC, linear regression and Bland \& Altman plots. Results. The Kinect assesses trunk compensations, hand range of motion, movement time and mean velocity with a moderate to excellent reliability. In contrast, elbow and shoulder range of motion, time to peak velocity and path length ratio have a poor to moderate reliability, indicating that these variables should be interpreted with caution. Finally, instantaneous hand and elbow tracking are not precise enough to reliably assess Cartesian and angular kinematics over time, rendering variables such as the number of velocity peaks and the peak hand velocity unusable. Conclusions. Thanks to its ease of use and markerless properties, the Kinect can be used in clinical routine for semi-automated quantitative diagnostics guiding individualised rehabilitation of the upper limb. However, engineers and therapists must bear in mind the limitations of the Kinect for the instantaneous tracking of the hand and elbow.

\section{Keywords}

Kinect - Reaching - Stroke - Rehabilitation - Upper limb - PANU - Kinematics - Markerless - Mocap - Motion capture

\section{1) Background}

Stroke results in major movement deficits, especially in the upper limb. To provide the best possible rehabilitation, therapists regularly assess the motor performance of individuals with stroke. However, the clinical scales used by therapists suffer from several limitations. First, clinical scales have a subjective scoring system which limits the reliability of ratings between therapists and over time [1]. Second, clinical scales are too often insensitive to changes. For example, in most of its items, the FuglMeyer Assessment only supports 3 rating levels $(0,1$ or 2$)$, whereas it is considered as one of the 
strongest clinical scales [2]. Third, most clinical scales do not sufficiently account for compensations that may occur in stroke movement $[3,4]$, which cancels out the differences between a true recovery and a compensation pattern [5].

To go beyond these limitations, scientists use motion capture to quantify the motor deficits. Indeed, upper limb and trunk kinematics are more sensitive to changes than clinical scales [6, 7], and can even predict motor outcomes over several months $[8,9]$. Moreover, motion capture makes compensation assessment easy and more objective $[3,10,11]$. Despite these advantages, kinematic assessment of the upper limb remains little used in clinical practice because of its poor feasibility. Indeed, motion capture systems are expensive and require a large volume to perform the movements. In addition, patients have to be suited up with markers placed with accuracy, which takes precious time in the clinical context. Finally, these technologies require a high technical level to extract valuable variables from raw data.

Since its release in 2013, the Kinect v2 (also known as Kinect One or Kinect for Xbox One, Microsoft, USA) has been widely used for rehabilitation purpose and has largely contributed to the rise of virtual reality in rehabilitation trials. Virtual reality with Kinect may be beneficial in improving upper limb function and activities of daily living when used as an adjunct to usual care (to increase overall therapy time) $[12,13]$. However, although the markerless and ease of use properties of the Kinect v2 facilitate its use in clinical routine, and its value for gait analysis has been documented [14], the validity of the Kinect for assessing upper limb kinematics after stroke remains to be tested.

Previous works shows that the Kinect $\mathrm{v} 2$ has an average accuracy of 10-15 mm but can generate distance errors up to $80 \mathrm{~mm}[15,16]$. In addition, the Kinect would detect range of motion (ROM) with 1 to $10^{\circ}$ error [17-20] but this result should be taken carefully given the variability between the studies. Indeed, some authors argue that the Kinect v2 has excellent reliability, especially for flexion of the elbow and shoulder [21], but others conclude that the kinematics obtained by the Kinect are unreliable [22] and that the use of IMUs should be preferred for motor assessment [23].

In people with stroke, when assessed by the Kinect v2, hand and trunk range of motion are valid and reliable [24] and kinematics can distinguish the reaching performance between healthy control, lessaffected side and more-affected side of patients with stroke [25-27]. Yet, there is no further information on precise kinematic variables.

The goal of our study is to investigate to what extent the Kinect $\mathbf{v 2}$ is valid and reliable for the kinematic assessment of reaching movements in people with stroke. To do so, we simultaneously recorded reaching movements with the Kinect $\mathrm{v} 2$ and the Vicon motion capture system considered as gold-standard. We hypothesized that the Kinect v2 will provide the same information as the Vicon system.

Because it was not possible to ask patients to come to the laboratory, we tested the reliability of the Kinect v2 with a model of stroke behaviour [28]. Specifically, we asked healthy participants to perform a series of reaching movements with their hand loaded to $75 \%$ of their maximum voluntary antigravity torque. In this loaded condition, healthy participants spontaneously develop compensations similar to those observed in most stroke patients, including trunk flexion and rotation, reduced shoulder abduction, and reduced movement speed. 


\section{2) Methods}

\subsection{Participants}

26 healthy participants (12 males, age $21 \pm 3$ years, 3 left-handed, height $1.73 \pm 0.09 \mathrm{~m}$, weight 66.92 $\pm 9.29 \mathrm{~kg}$ ) took part to this study.

Participants were not included if they had shoulder pain or any other problem that could affect their movement. This study was performed in accordance with the 1964 Declaration of Helsinki. The local ethics committee approved the study (IRB-EM 1901C).

\subsection{Experimental protocol}

Participants performed the PANU test [3] as described in a previous study [28]. Participants had to reach a target with the side of their thumb nail. The target was a table tennis ball fixed in front of the participant at a height of $0.80 \mathrm{~m}$, just within the anatomical reaching distance for the hand. The starting position was seated, feet on the ground, back in contact with the chair and forearms on the armrests.

Participants completed 5 reaches both in the spontaneous trunk use condition and in the restrained trunk use condition. In the spontaneous trunk use condition, participants had to reach the target at a natural pace, wait 1 second and return to the starting position. In the restrained trunk use condition, participants had to reach the target while minimising trunk movement: the experimenter manually applied a light proprioceptive feedback on the participant's shoulders, as a reminder to minimise trunk movement. We did not use a belt to restrain the trunk in order to leave the participant free to use the trunk if necessary, and thus avoid task failure.

The assessed hand was chosen pseudo-randomly (12 left, 14 right) so that half of the participants performed the task with their dominant hand, and the other half with their non-dominant hand. The weight of the arm including the dumbbell was set to $75.0 \%$ ( $\pm 5.5 \%$ ) of the maximum antigravity force (MAF) in the posture with the hand at the target.

\subsection{Experimental setup}

The movements of the participants were recorded by both a Vicon motion capture system and a Kinect v2. The data obtained by the Kinect were then compared to the data obtained by the Vicon, the latter being considered as the ground truth.

\subsubsection{Vicon sensor}

The Vicon system (Oxford Metrics, U.K.) is a marker-based optoelectronic motion capture tool that is widely used for kinematic measurements [29]. Indeed, with a similar setting to ours, the error of the Vicon is $0.15 \mathrm{~mm} \pm 0.025 \mathrm{~mm}$ in static and remains less than $2 \mathrm{~mm}$ in dynamic [30]. In this study, we used a 6-camera rectangle Vicon system with a sampling frequency of $100 \mathrm{~Hz}$. Vicon time series were recorded using "Vicon Nexus 2" software (Oxford Metrics, U.K.).

\subsubsection{Kinect sensor}

The Kinect v2 (Microsoft, USA) is a markerless motion capture tool combining 3 sensors (a RGB colour camera, a depth sensor and an infrared sensor) to provide the 3D position of 25 landmarks on a skeleton with a sampling rate of $30 \mathrm{~Hz}$ [24]. The Kinect was connected to a PC running the "MaCoKi" software (NaturalPad, Montpellier, France) developed from the Kinect SDK (v2.0_1409, Microsoft, USA) to record the position time-series of the hands and trunk. As recommended in previous studies, we placed the Kinect in front of the participant, at a distance of $1.50 \mathrm{~m}$, a height of $1.40 \mathrm{~m}$ and with no direct sunlight to minimise errors [18, 31-33] (Fig. 1). 


\subsubsection{Position of landmarks for the Vicon and for the Kinect}

In order to compare the Kinect data to the Vicon data, we placed the Vicon markers as close as possible to the joint centers located by the Kinect. Thus, we placed markers at the manubrium (spine-shoulder for Kinect), and for each body side on the 1st metacarpal (wrist for Kinect), the lateral epicondyle of the humerus (elbow for Kinect), the acromion process (shoulder for Kinect), and the anteriosuperior iliac spine. For each side, we corrected the anteriosuperior iliac spine marker position before data analysis to best match the anatomical center of hips joints (hips for Kinect). In order to facilitate the reading, the spine shoulder marker is renamed "trunk marker" in the text.

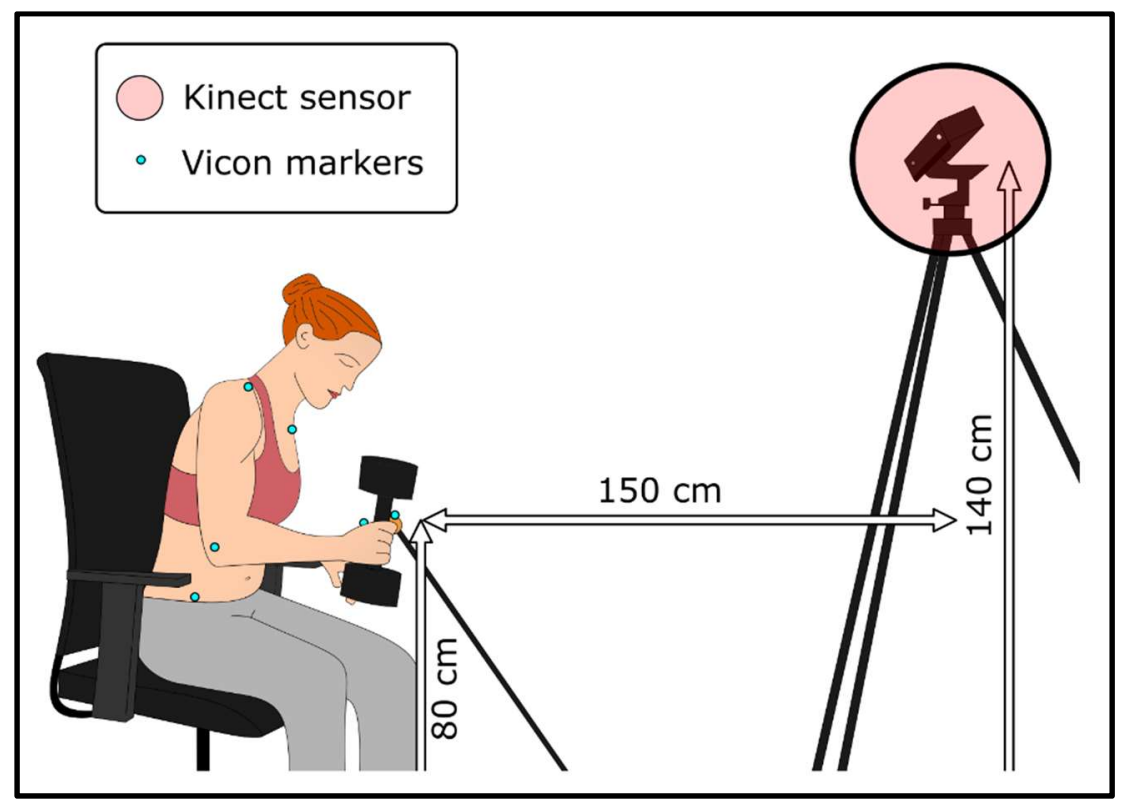

Figure 1. Experimental setup. 3D upper limb kinematics were simultaneously recorded by the Kinect and the Vicon motion capture systems. Vicon markers were placed on the target, hands, elbows, shoulders, manubrium and hips of the participant as close as possible to the joint centres located by the Kinect. The Kinect was located in front of the participant, at a distance of $1.50 \mathrm{~m}$ and a height of $1.40 \mathrm{~m}$. The target (orange table tennis ball) was located at a height of $0.80 \mathrm{~m}$, just within the anatomical reaching distance for the hand.

\subsection{Data processing}

Data processing was performed with SciLab 6.0.2. The Kinect being positioned obliquely to the ground to minimise visual occlusions of the elbow, we realigned the Kinect axes to match the Vicon axes. This realignment was performed using a solid transformation of the Kinect data based on the "LeastSquares Fitting" method [34] implemented in a matlab function by Nghia Ho [35].

All position time series were low pass filtered at $2.5 \mathrm{~Hz}$ with a dual pass second order Butterworth filter. We choose a cut-off frequency of $2.5 \mathrm{~Hz}$ because the analysis of raw data showed that the frequency band $0-2.5 \mathrm{~Hz}$ contains at least $95 \%$ of the spectral density of the time series data.

We first calculated the start and end of each reaching movement in the one-dimensional task space [28]. Because the goal of a reaching task is to bring the hand to the target, that is, to reduce the handto-target distance, what is important for task success is the hand-to-target Euclidean distance. The hand-to-target Euclidean distance summarises the 3D effector space into a 1D task space (where movement matters) leaving aside a $2 \mathrm{D}$ null space (where movement does not impact task success). We fixed the beginning of the movement (t0) when the Euclidean velocity of the hand in task space became positive and remained positive until the maximum velocity. The end of the movement (tfinal) was when the Euclidean distance to the target reached its minimum. 
Angles presented in this study were calculated as the difference between the anatomical angle at tfinal and the anatomical angle at t0, as described in the equation 1.

$$
\text { retained angle }=\text { anatomical angle } \text { tfinal }_{-} \text {anatomical angle } \text { to }_{0}
$$

\subsection{Statistical analysis}

We assessed the degree of reliability between the Kinect and the Vicon variables using intraclass coefficient correlation (ICC) and coefficient of determination $\left(r^{2}\right)$. We complemented these measures with Bland and Altman plots to evaluate the validity of the Kinect through the difference in means and to estimate an agreement interval through the $95 \%$ limits of agreement [36]. To compare validity across variables, a relative systematic error was calculated as error relative $=\frac{\text { mean }_{\text {vicon }}-\text { me }_{\text {kinect }}}{\text { mean }_{\text {vicon }}}$.

ICC estimates were calculated using $R$ (version 3.6.1) based on a single-rating, consistency, one-way random-effect model. As stated by Koo \& Li, "values less than 0.5 are indicative of poor reliability, values between 0.5 and 0.75 indicate moderate reliability, values between 0.75 and 0.9 indicate good reliability, and values greater than 0.90 indicate excellent reliability" [37]. We used the same limits for

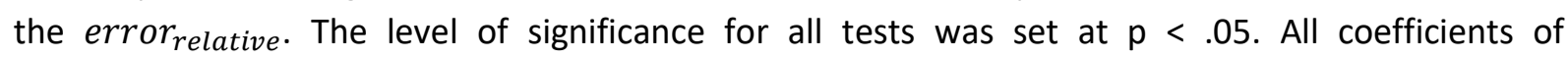
determination $r^{2}$ were found to be statistically significant.

\section{3) Results}

The results are reported in table 1. 
Table 1. Reliability and validity of the Kinect main kinematic variables used in the analysis of reaching in stroke [38]. Reliability measures the consistency of the results compared to the ground truth (i.e., for each individual, how close the Kinect measure is to the ground truth). A perfect reliability between Kinect and Vicon data would result in an intraclass correlation coefficient (ICC) of 1 and a coefficient of determination $\left(r^{2}\right)$ of 1 as well. The reliability of each of the main variables is summarised on the $Y$ axis of the figure 6 using the ICC. Validity measures the extent to which the results are close to the ground truth on average (i.e., the higher the percentage of error on average, the lower the validity). A perfect validity would result in a difference in means of 0 in the Bland and Altman plot, and thus in a relative systematic error of 0 . The validity of each of the main variables is summarised on the $X$ axis of the figure 6 using the relative systematic error.

\begin{tabular}{|c|c|c|c|c|c|c|c|c|c|}
\hline \multirow{2}{*}{\multicolumn{2}{|c|}{ Variable }} & \multicolumn{3}{|c|}{ Reliability } & \multicolumn{4}{|c|}{ Validity } & Figure \\
\hline & & $\begin{array}{l}\text { Degree of } \\
\text { reliability }\end{array}$ & ICC & $r^{2}$ & $\begin{array}{l}\text { Degree of } \\
\text { validity }\end{array}$ & $\begin{array}{c}\text { Direction of } \\
\text { systematic error }\end{array}$ & $\begin{array}{c}\text { Absolute } \\
\text { Systematic error } \\
\text { (Bias) }\end{array}$ & $\begin{array}{c}\text { Relative } \\
\text { Systematic error }\end{array}$ & \\
\hline \multirow{6}{*}{ Angles } & $\begin{array}{c}\text { Elbow } \\
\text { extension }\end{array}$ & Moderate & .55 & .56 & Poor & Overestimation & $+13.95^{\circ}( \pm 15.67)$ & $+62.5 \%( \pm 70.2)$ & Fig. 2 - Top panel \\
\hline & $\begin{array}{l}\text { Shoulder } \\
\text { abduction }\end{array}$ & Poor & .29 & .13 & Good & Overestimation & $+2.67^{\circ}( \pm 11.44)$ & $+22.0 \%( \pm 94.7)$ & Fig 2 - Middle panel \\
\hline & Shoulder flexion & Moderate & .50 & .37 & Good & Overestimation & $+6.61^{\circ}( \pm 11.37)$ & $+19.4 \%( \pm 33.4)$ & Fig 2 - Bottom panel \\
\hline & $\begin{array}{c}\text { Trunk anterior } \\
\text { flexion }\end{array}$ & Good & .82 & .73 & Moderate & Underestimation & $-1.22^{\circ}( \pm 3.05)$ & $-35.3 \%( \pm 88.9)$ & Fig. 3 - Top panel \\
\hline & Trunk rotation & Poor & .38 & .25 & Moderate & Underestimation & $-4.10^{\circ}( \pm 8.22)$ & $-25.7 \%( \pm 51.6)$ & Fig. 3 - Bottom panel \\
\hline & Movement time & Good & .76 & .58 & Excellent & Overestimation & $+0.01 \mathrm{~s}( \pm 0.41)$ & $+0.4 \%( \pm 17.6)$ & Fig. 4 - Top panel \\
\hline Efficiency & $\begin{array}{l}\text { Path length } \\
\text { ratio }\end{array}$ & Moderate & .51 & .48 & Excellent & Overestimation & $+0.11( \pm 0.14)$ & $+9.2 \%( \pm 11.7)$ & $\begin{array}{l}\text { Fig. } 4 \text { - Middle } \\
\text { top panel }\end{array}$ \\
\hline Planning & $\begin{array}{c}\text { Time to peak } \\
\text { velocity }\end{array}$ & Moderate & .55 & .32 & Excellent & Underestimation & $-0.05 s( \pm 0.34)$ & $-5.0 \%( \pm 33.7)$ & $\begin{array}{l}\text { Fig. } 4 \text { - Middle } \\
\text { bottom panel }\end{array}$ \\
\hline Smoothness & $\begin{array}{c}\text { Number of } \\
\text { velocity peaks }\end{array}$ & Poor & .38 & .20 & Good & Overestimation & $+0.84( \pm 1.86)$ & $+24.0 \%( \pm 53.8)$ & Fig. 4 - Bottom panel \\
\hline \multirow{2}{*}{ Speed } & Peak velocity & Poor & .21 & .11 & Good & Overestimation & $\begin{array}{c}63.04 \mathrm{~mm} \cdot \mathrm{s}^{-1}( \pm \\
194.87)\end{array}$ & $+15.5 \%( \pm 47.9)$ & Fig. 5 - Top panel \\
\hline & Mean velocity & Moderate & .70 & .53 & Excellent & Overestimation & $\begin{array}{c}+4.91 \mathrm{~mm} \cdot \mathrm{s}^{-1}( \pm \\
40.10)\end{array}$ & $+2.7 \%( \pm 21.7)$ & Fig 5 - Bottom panel \\
\hline \multirow{6}{*}{ Displacements } & PANU & Excellent & .94 & .92 & Good & Overestimation & $+1.88 \%( \pm 4.43)$ & $+12.3 \%( \pm 28.7)$ & Fig. Supp. - $1^{\text {st }}$ panel \\
\hline & PAU & Excellent & .95 & .96 & Excellent & Underestimation & $-2.88 \%( \pm 3.65)$ & $-3.1 \%( \pm 4.0)$ & Fig. Supp. - $2^{\text {nd }}$ panel \\
\hline & $\Delta$ Trunk & Excellent & .93 & .95 & Good & Underestimation & $-15.04 \mathrm{~mm}( \pm 12.72)$ & $-21.5 \%( \pm 18.2)$ & Fig. Supp. - $3^{\text {rd }}$ panel \\
\hline & $\Delta$ Shoulder & Good & .88 & .86 & Good & Underestimation & $-22.26 \mathrm{~mm}( \pm 26.75)$ & $-20.0 \%( \pm 24.0)$ & Fig. Supp. $-4^{\text {th }}$ panel \\
\hline & $\Delta$ Elbow & Poor & .47 & .37 & Good & Underestimation & $-34.29 \mathrm{~mm}( \pm 50.61)$ & $-11.9 \%( \pm 17.5)$ & Fig. Supp. $-5^{\text {th }}$ panel \\
\hline & $\Delta$ Hand & Moderate & .71 & .73 & Excellent & Overestimation & $+28.40 \mathrm{~mm}( \pm 28.70)$ & $+8.4 \%( \pm 8.5)$ & Fig. Supp. $-6^{\text {th }}$ panel \\
\hline
\end{tabular}


bioRxiv preprint doi: https://doi.org/10.1101/2022.01.18.476737; this version posted January 20, 2022. The copyright holder for this preprint (which was not certified by peer review) is the author/funder, who has granted bioRxiv a license to display the preprint in perpetuity. It is made available under aCC-BY-NC-ND 4.0 International license.

\subsection{Angles}
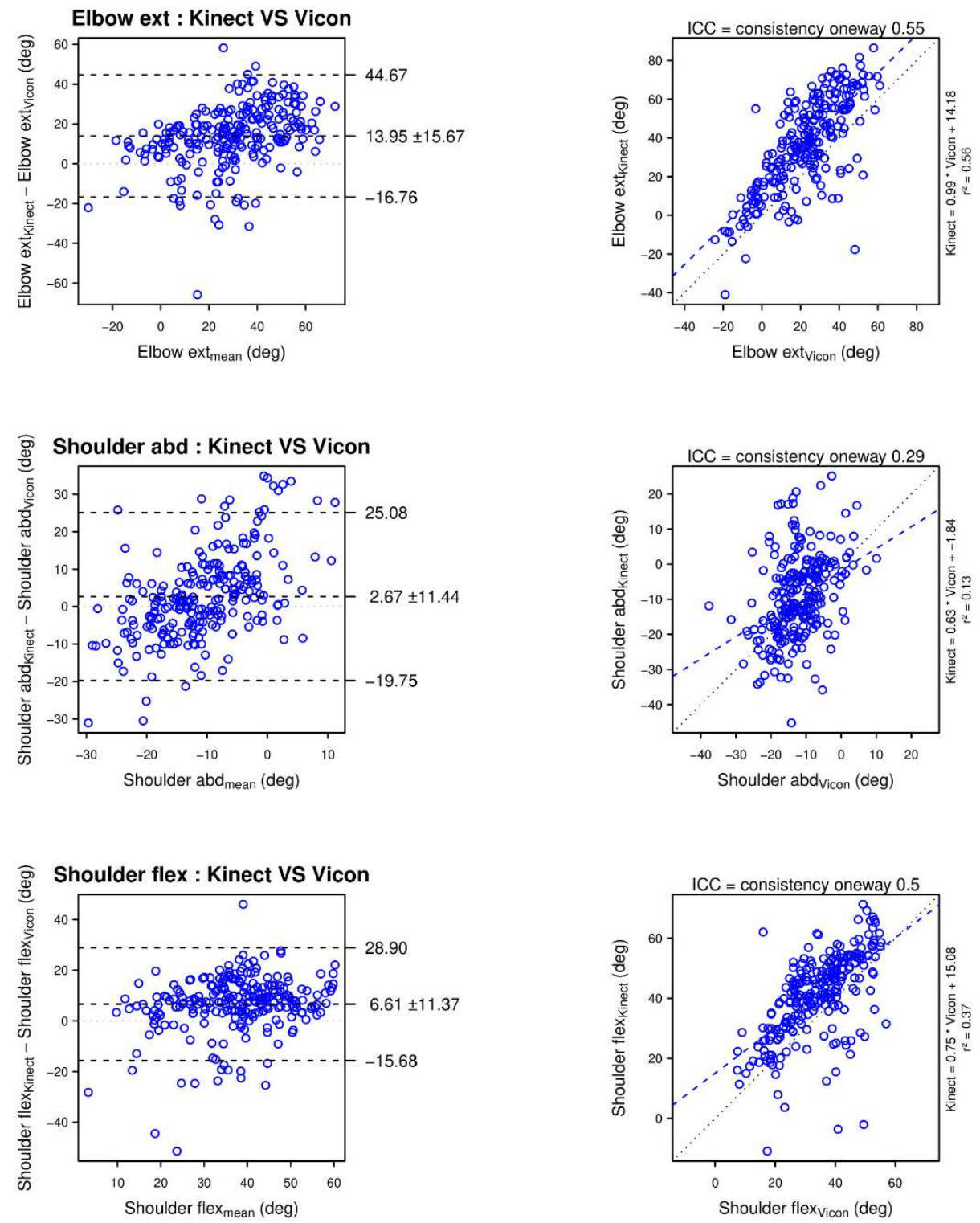

Figure 2. Comparison of the arm and shoulder angles assessed with the Kinect and the Vicon systems. Panels in the first row illustrate elbow extension (Elbow ext). Panels in the second row illustrate shoulder abduction (Shoulder abd). Panels in the third row illustrate shoulder flexion (Shoulder flex). For each row, the left panel represents the Bland and Altman plot and the right panel represents the linear regression plot. When assessed with the Kinect, elbow extension is moderately reliable but highly overestimated, shoulder abduction is poorly reliable and slightly overestimated in average, shoulder flexion is moderately reliable and slightly overestimated. 
bioRxiv preprint doi: https://doi.org/10.1101/2022.01.18.476737; this version posted January 20, 2022. The copyright holder for this preprint (which was not certified by peer review) is the author/funder, who has granted bioRxiv a license to display the preprint in perpetuity. It is made available under aCC-BY-NC-ND 4.0 International license.
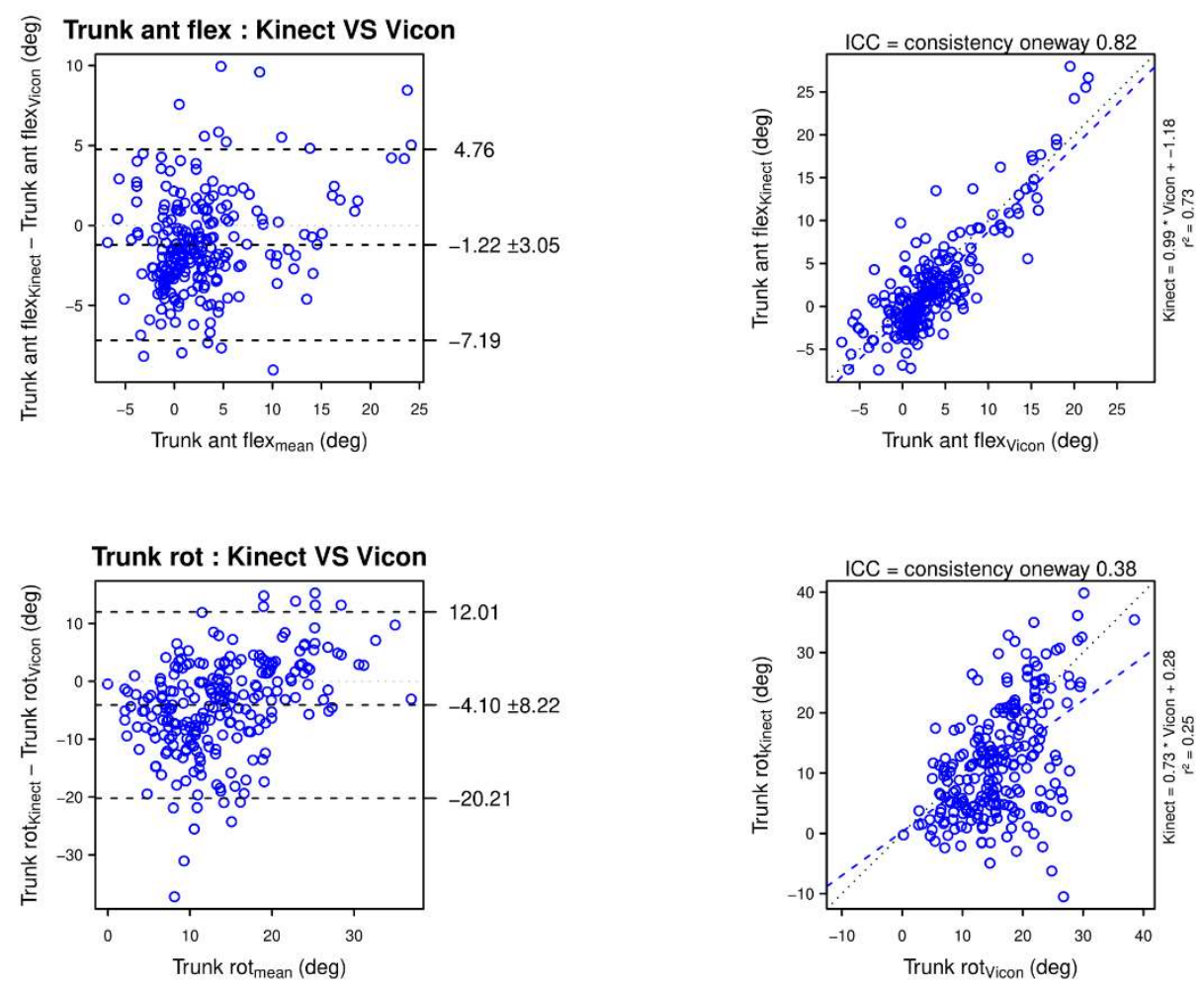

Figure 3. Comparison of the trunk angles assessed with the Kinect and the Vicon systems. Panels in the first row illustrate trunk anterior flexion (Trunk ant flex). Panels in the second row illustrate trunk rotation (Trunk rot). For each row, the left panel represents the Bland and Altman plot and the right panel represents the linear regression plot. When assessed with the Kinect, trunk anterior flexion is goodly reliable but moderately underestimated, trunk rotation is poorly reliable and moderately underestimated. 
bioRxiv preprint doi: https://doi.org/10.1101/2022.01.18.476737; this version posted January 20, 2022. The copyright holder for this preprint (which was not certified by peer review) is the author/funder, who has granted bioRxiv a license to display the preprint in perpetuity. It is made available under ACC-BY-NC-ND 4.0 International license.

\subsection{Efficiency, planning and smoothness}
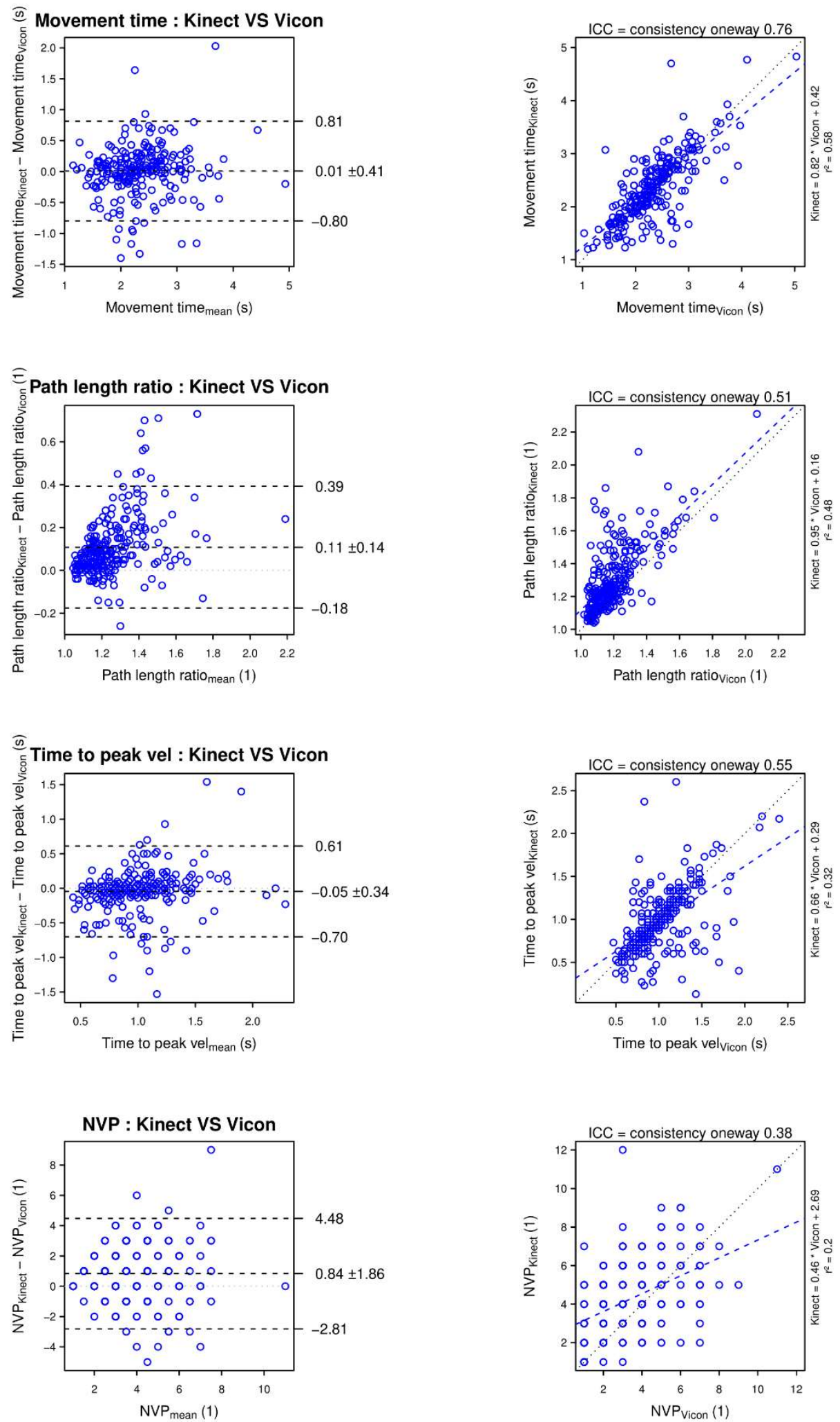

Figure 4. Comparison of hand efficiency, planning and smoothness assessed with the Kinect and the Vicon systems. Panels in the first row illustrate movement time (Movement time). Panels in the second row illustrate hand path length ratio (Path length ratio). Panels in the third row illustrate time to peak hand velocity (Time to peak vel). Panels in the fourth row illustrate number of hand velocity peaks (NVP). For each row, the left panel represents the Bland and Altman plot and the right panel represents the linear regression plot. When assessed with the Kinect, movement time is goodly reliable and have quasi no systematic error in average, path length ratio is moderately reliable and very slightly overestimated in average, time to peak 
velocity is moderately reliable and very slightly underestimated, number of velocity peaks is poorly reliable and slightly overestimated.

\subsection{Speed}
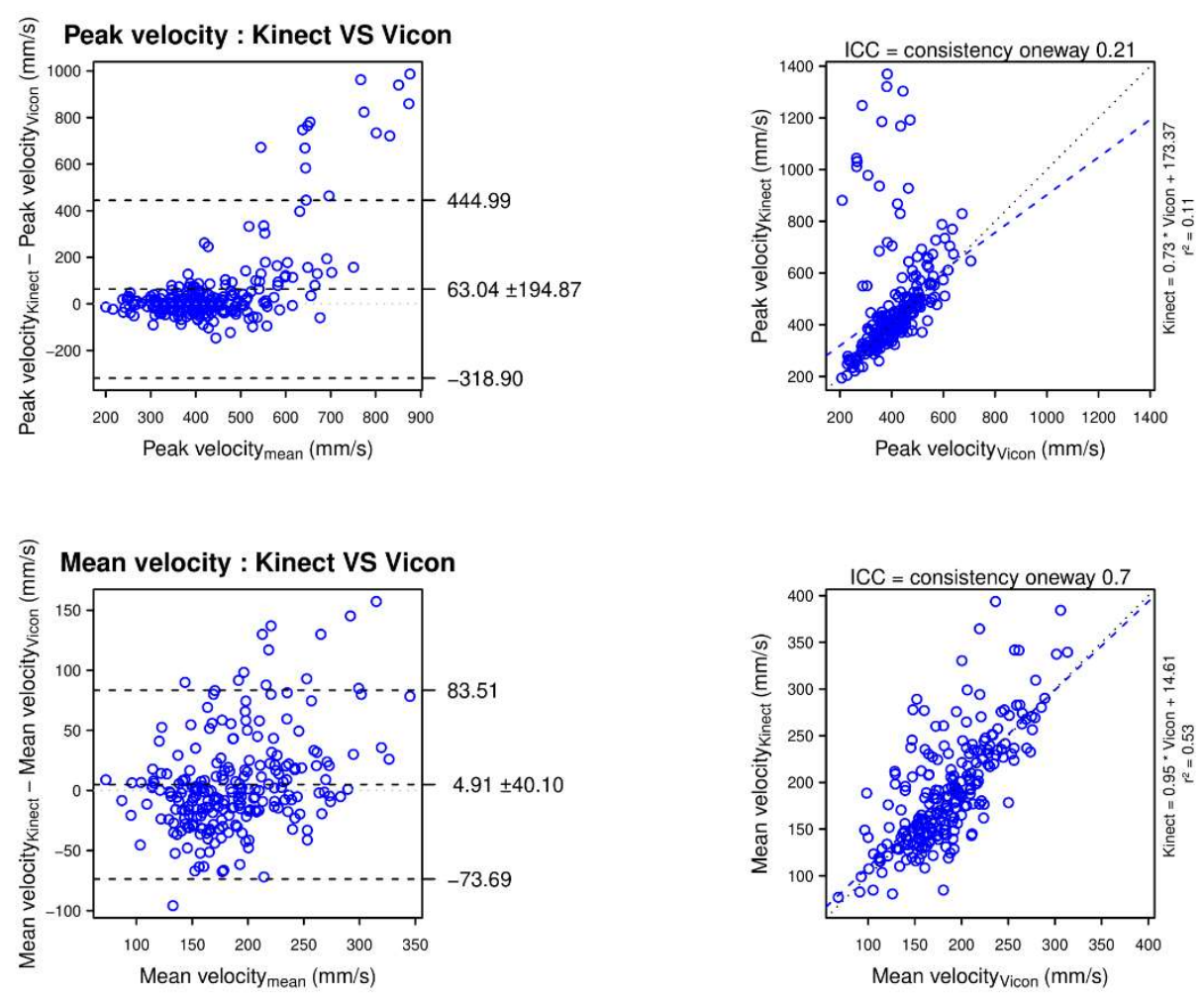

Figure 5. Comparison of the hand speed assessed with the Kinect and the Vicon systems. Panels in the first row illustrate peak hand velocity (Peak velocity). Panels in the second row illustrate mean hand velocity (Mean velocity). For each row, the left panel represents the Bland and Altman plot and the right panel represents the linear regression plot. When assessed with the Kinect, peak velocity is poorly reliable and slightly overestimated, mean velocity is moderately reliable and very slightly overestimated.

\subsection{Displacements}

The figure of displacements is in the supplementary data.

\section{4) Discussion}

Our study shows that in a horizontal reaching task, the Kinect measures trunk forward compensations with a good to excellent reliability and validity, but is unsensitive to low amplitude trunk rotation. The Kinect also measures hand and trunk range of motion as well as movement time and mean hand velocity with a moderate to good reliability, and with a good to excellent validity.

In contrast, the Kinect assesses variables involving elbow extension, shoulder flexion and shoulder abduction with a poor to moderate reliability and overall overestimates the variables. Finally, instantaneous Cartesian and angular measures with the Kinect are not precise enough which artificially creates jerky movements and overestimates NVP, Path Length Ratio, and Peak Velocity. Time to Peak Velocity is also affected resulting in a moderate reliability. Main results are summarised in Figure 6.

These data indicate that, although the Kinect is not reliable enough to analyse fine kinematics over time, the Kinect does allow for global motion analysis (such as range of motion, movement time and mean velocity) with sufficient reliability in a clinical context. 


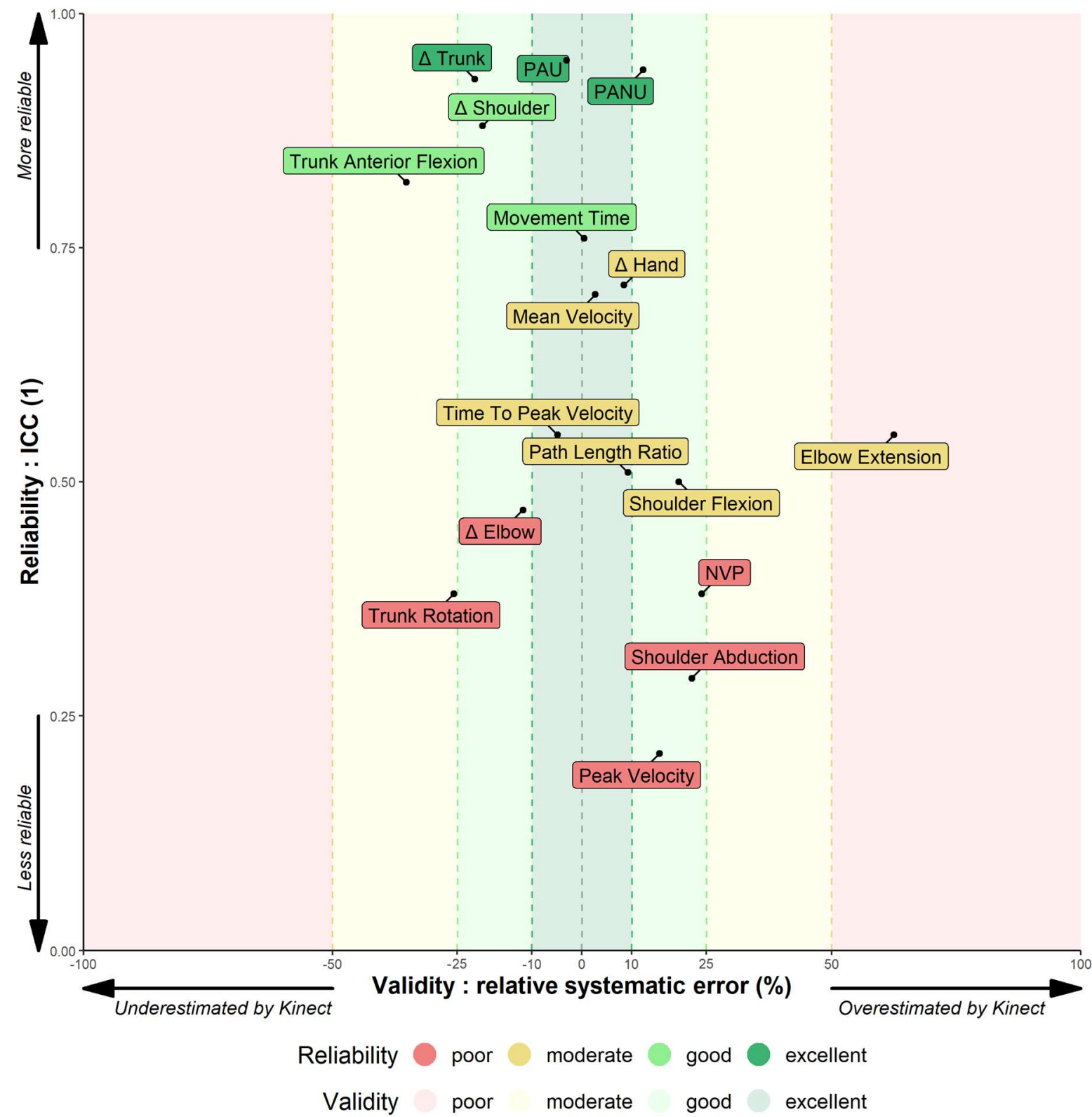

Figure 6. Summary of validity and reliability of 17 kinematic variables assessed by the Kinect. The X axis represents the validity through the relative error. The $Y$ axis represents the reliability through the one-way ICC. The closer the variable is to the centre on the $X$ axis, the more valid it is. The higher the variable is on the $Y$ axis, the more reliable it is. A perfect match between the Kinect and the Vicon values would place the variable at the centre on the $X$ axis (error $=0$ ) and at the top on the $Y$ axis (ICC = 1). The figure shows that averaged postural and angular measurements are much more reliable than instantaneous Cartesian measures.

\subsection{Validation of the kinematic assessment obtained by Kinect}

The aim of the study was to assess the reliability and validity of the Kinect main kinematic variables used in the analysis of reaching in stroke [38].

\section{Trunk motion}

The Kinect has excellent reliability on trunk displacement $(I C C=.93)$ and good reliability on shoulder displacement $(I C C=.88)$ and anterior flexion of the trunk (ICC $=. .82)$. Because of a low mean trunk flexion $\left(3.43^{\circ}\right)$, the difference between means of $-1.22 \pm 3.05^{\circ}$ overrepresent the error relative $(-35 \%)$ in the figure 6 . 
In contrast, trunk rotation was poorly assessed with the Kinect (ICC $=.38)$, in particular with an underestimation of up to $20^{\circ}$ of low trunk rotation (Fig. 3, top panel, left column) and other authors found the same result [18]. Note that the Vicon might also overestimate the low values of trunk rotation due to soft tissue artefact [39]. Indeed, trunk rotation being assessed mainly with the displacement of the shoulder marker on a transversal plane, a slight forward shift of the shoulder marker due to a shoulder flexion might artificially count for a trunk rotation. In any case, we propose that trunk rotation assessed with the Kinect should not be interpreted unless it exceeds $20^{\circ}$.

\section{Hand motion}

At the beginning of the reach, the Kinect sometimes confuses the forearm with the armrest. The Kinect correctly relocates the hand during the movement, creating a jerky correction. To a lesser extent, this temporo-spatial uncertainty occurs several times during the reach which artificially creates a jerkier movement, and overestimates the NVP and the path length ratio (Fig. $4,2^{\text {nd }}$ and $4^{\text {th }}$ panel). By suddenly modifying the hand position, the Kinect creates a local velocity peak, possibly resulting in an overestimation of the peak hand velocity (Fig. 5, top panel). Due to its relation to peak velocity, the time to peak velocity is also affected in some cases (Fig. 4, $3^{\text {rd }}$ panel). By averaging the velocity over the entire movement, the mean velocity resists these local uncertainties (Fig. 5, bottom panel).

Because the reliability of $\Delta$ hand and $\Delta$ trunk assessment are good to excellent, variables derived from the $\Delta$ hand and $\Delta$ trunk measures such as PAU and PANU also have an excellent reliability, which confirms our previous findings [24].

\section{The problem of elbow in a seated reaching task}

First, the elbow is often confused with the back part of the armrest, resulting in a backward shift of the actual elbow position at the beginning of the reach. Second, due to the position of the hand located between the Kinect and the elbow, elbow occlusion can occur leading to an error in the elbow position. Third, elbow is poorly located on a fully extended arm (such as at the end of the reach) because of the alignment forearm - upper arm. For these reasons, $\Delta$ elbow and side variables such as elbow extension, shoulder flexion and shoulder abduction are only poorly to moderately reliable and should be interpreted with caution.

To reduce elbow occlusion, some authors suggest installing the Kinect higher [18], in front of the participant $[32,33]$ and to perform functional movements in sitting $[18,40]$, but the present study shows that this is not enough to achieve sufficient precision for clinical interpretation of some variables. Other authors suggest installing the Kinect on the ipsilateral side of the movement with an angle of 30 to $45^{\circ}$ [41], and moving the Kinect when assessing the other side, but this make the experiment more complicated and therefore reduces the fast and ease of use characteristic of the Kinect.

\subsection{Smoothing out Kinect errors}

The present study shows that the occlusion of the elbow and the confusion between the forearm and the armrest produce large errors resulting in a jerky movement. A first solution might be to use a chair with small armrests to decrease confusion errors. Furthermore, a correct filtering should be applied to the raw data. In fact, the analysis of raw data showed that the frequency band $0-2.5 \mathrm{~Hz}$ contains $95 \%$ of the spectral density. Applying a $2^{\text {nd }}$ order Butterworth type filtering with a cutoff frequency of 2.5 $\mathrm{Hz}$ greatly improved cartesian kinematics (table 2), while it had no effect on other variables (ICC changes $\leq .03$ ). The ideal cut-off frequency for individuals with stroke might be higher than $2.5 \mathrm{~Hz}$ since people with stroke have more segmented movements than healthy individuals $[42,43]$. Thus, a too low cut-off frequency might remove important information about the movement. However, our study 
shows that range of motion and averaged variables do not suffer from a lack of filtering, and can still be interpreted without filtering.

Table 2. Reliability improvements through filtering. The filtering consisted of applying a $2^{\text {nd }}$ order Butterworth filter with a cutoff frequency of $2.5 \mathrm{~Hz}$ on raw time series data. All variables shown in the table improved from filtering (ICC changes $\geq .12$ ). Variables not shown in this table did not benefit from filtering (ICC changes $\leq .03$ ).

\begin{tabular}{|c|c|c|c|c|c|}
\hline & $\begin{array}{c}\text { Movement } \\
\text { time }\end{array}$ & $\begin{array}{c}\text { Path length } \\
\text { ratio }\end{array}$ & $\begin{array}{c}\text { Time to peak } \\
\text { velocity }\end{array}$ & NVP & Peak velocity \\
\hline $\begin{array}{c}\text { ICC before } \\
\text { filtering }\end{array}$ & .64 & -.12 & .19 & -.30 & -.05 \\
\hline $\begin{array}{c}\text { ICC after } \\
\text { filtering }\end{array}$ & .76 & .51 & .55 & .38 & .21 \\
\hline
\end{tabular}

Other authors explored solutions to reduce Kinect errors. A deep learning algorithm applied to time series data reduced Kinect errors on shoulder and elbow range of motion by $88.8 \pm 12.4 \%$ [44]. Another approach is to fusion the data of several Kinects to minimise occlusions and optimise limb tracking [45-47]. Ryselis reported an overall increase in accuracy of $15.7 \%$. A combination of a Kinect and several Inertial Measurement Units (IMUs) could also be used to reduce the upper limb position error up to $20 \%$ according to Jatesiktat et al. [48-50]. Finally, a device-independent approach is to incorporate body constraints (such as human skeleton length conservation and temporal constraints) to enhance the continuity of the estimated skeleton [51]. These solutions have the potential to consequently improve the accuracy of the Kinect while remaining affordable, even if the solutions require a high technical level and might lengthens the duration of patient preparation.

\subsection{Kinematic assessment of the upper limb in clinical routine for personalised rehabilitation post-stroke}

\section{Markerless motion capture advantages for kinematic assessment}

Due to its ease of use and markerless characteristic, the Kinect allows therapists to perform a simple kinematic assessment of the upper limb in about 5 minutes [3]. The software development kit (SDK) of the Kinect being openly available, the development of a software that automatically clean the data and compute the valuable kinematic variables is also possible. In addition, if the rehabilitation department does not already use markerless motion capture for virtual reality rehabilitation, the low cost of such devices ensures their accessibility.

\section{Kinematic assessment to better understand the level of motor recovery of the patient}

A seated reaching task with and without trunk restraint gives the therapist valuable information to better understand arm-trunk use [3], but the very same task, when recorded with the Kinect, opens the door to a more comprehensive kinematic assessment.

The movement time, the mean hand velocity, the path length ratio and the time to peak velocity reflect the spatial and temporal efficiency of the movement. A low mean hand velocity or a low time to peak velocity when combined to a high path length ratio or a high NVP reflect the increased importance of feedback and corrections during the reach and therefore signal a decreased efficiency of open-loop control $[52,53]$. Except for the NVP, the Kinect measures these kinematic outcomes with acceptable accuracy-reliability (table 1), making the Kinect a valuable tool for monitoring changes in upper extremity kinematics over rehabilitation.

The elbow, shoulder and trunk range of motion describe the motor strategy and quantify the level of compensation used by the patient. Reduced elbow extension and shoulder flexion signal a deficit in 
upper limb movement that is often compensated by an increase of trunk flexion and trunk rotation, and a freeze of shoulder adduction [10,54]. The Kinect measures these kinematic outcomes with varied accuracy-reliability making the Kinect a valuable tool for monitoring trunk flexion, but not for monitoring shoulder and elbow movements.

Quantifying the non-use of the shoulder-elbow joints with the PANU score [3] informs about what the patient can do, but does not do spontaneously. If the patient compensates with the trunk but can also perform the movement without using the trunk (PANU score $>6.5 \%$ ), then at least some of the compensation is not mandatory to succeed in the task. In contrast, if the patient has compensation and is unable to perform the movement without this compensation (PANU score $<6.5 \%$ ), then compensation is mandatory to succeed in the task [3]. This distinction is important because a compensation that improves reach efficiency should not be considered the same as a compensation which does not improve reach efficiency. The Kinect measures the PANU score with excellent accuracyreliability (table 1), which confirms previous results [24].

\section{Early and regular kinematic assessment to individualise rehabilitation, enhance motivation and improve recovery}

Because kinematic monitoring is more sensitive to changes over the course of rehabilitation than clinical scales $[6,7]$, this could allow specific therapy to be planned based on specific changes in kinematics. For example, therapy focused on arm use makes sense for patients with high PANU scores but is certainly less suitable for patients with mandatory compensations.

Therapists can also provide kinematic feedback to patients and set goals to involve them in rehabilitation. Fine-tuned feedback enhances motivation and increases the level of acceptance of treatment by patients [55]. Thus, providing kinematic feedback to patients leads to better recovery $[56,57]$.

\subsection{Limitations of the study}

This study faces several limitations. The experiment was conducted on healthy volunteers whose movement characteristics were experimentally manipulated to approximate those of people who have suffered a stroke. Though it is reassuring that we here replicate the main results obtained with a Kinect and with patients [24], the Kinect-Vicon comparison with patients with stroke is a necessary additional step to go beyond the basic results presented here. Moreover, to induce a stroke-like movement, we asked healthy participants to hold a dumbbell during the reach. The presence of the dumbbell in the hand could have affected the ability of the Kinect to correctly locate the hand, even though this effect is likely small (i.e., the endpoint accuracy is similar for the loaded and unloaded hand and no significant difference was detected in the hand velocity root-mean-square error (RMSEhand) between conditions). The dumbbell could also have induced a greater occlusion of the elbow, which might have affected the performance of the elbow tracking, as evidenced by an increased error in the RMSE $\mathrm{E}_{\text {elbow }}$ of $40 \pm 72 \%$ between the unload and load conditions. However, this increased error in the elbow tracking is compensated by a wider dispersion in the load condition, and thus no improvement in the validity and reliability of the variables shown in this study was found in the unload condition. Based on the results presented here, it is effective to clinically assess upper limb kinematics after stroke with the Kinect v2, and this should be confirmed with a forthcoming clinical trial (https://clinicaltrials.gov/ct2/show/NCT04747587). Finally, the results presented here are only relevant for the same type of task, which is a unilateral horizontal seated reaching task. Results might differ in another type of task, such as non-horizontal reaching, or finger to noise test [58]. Indeed, due to the uncertainty of the depth measurement with the Kinect [15], movements in the frontal plane 
bioRxiv preprint doi: https://doi.org/10.1101/2022.01.18.476737; this version posted January 20, 2022. The copyright holder for this preprint (which was not certified by peer review) is the author/funder, who has granted bioRxiv a license to display the preprint in perpetuity. It is made available under aCC-BY-NC-ND 4.0 International license.

that are less dependent on depth changes might show a better accuracy than movements with variable depth when measured with the Kinect.

\subsection{Future work}

There is a need to replicate the validation in a population with movement disorders, whether for a Kinect or for any other markerless system with similar benefits. In addition, as Kinect-like systems are increasingly used in virtual reality rehabilitation to monitor the recovery process [13, 59], replication should focus on a wide variety of movements such as those used in virtual reality rehabilitation. Indeed, the present study assessed the validity of kinematic variables in a horizontal seated reaching task, but virtual reality protocols cover a much wider range of variables and tasks.

However, although Kinect is still widely used in virtual reality rehabilitation, Microsoft has stopped selling the Kinect since 2017. Instead, with the emergence of machine learning for image processing, many low-cost 2D joints tracking come to light [60], which led to 3D motion reconstruction modules based on multiple RGB cameras and joint triangulation as provided by OpenPose [61], VideoPose3D [62] or Learnable Triangulation [63]. Microsoft has also aligned itself with the release of the Kinect Azure in 2020, a new version of the Kinect that uses a deep neural network (DNN) method instead of the random forest method used by the Kinect v2 [64]. In order to anticipate their growing use, future work should assess the feasibility, validity and reliability of these systems in a clinical or home environment.

\section{5) Conclusions}

The aim of this study was to assess the validity and reliability of the Kinect v2 for quantifying upper body kinematics with application to monitoring upper limb function after stroke. Although therapists should be mindful of the Kinect limitations, particularly with respect to instantaneous kinematics, our results show that the Kinect quantifies reaching efficiency, compensation and nonuse with sufficient reliability to be used for rehabilitation purpose. The quantitative variables that are adequately monitored by the Kinect can effectively supplement the clinical scales used by therapists. In addition, a periodic assessment of the deficit can allow precise longitudinal follow-up of motor recovery, which could improve the evaluation of the rehabilitation modalities and help to optimise the therapeutic pathway of patients. A final advantage of using lightweight markerless motion capture devices in clinical routine is that an accumulation of kinematic data could allow ambitious retrospective studies to be carried out in the long run.

Supplementary figure 1 legend. Comparison of nonuse and joint displacements assessed with the Kinect and the Vicon systems. Panels in the first row illustrate proximal arm nonuse (PANU). Panels in the second row illustrate proximal arm use (PAU). Panels in the third row illustrate trunk displacement ( $\triangle$ Trunk). Panels in the fourth row illustrate shoulder displacement ( $\Delta$ shoulder). Panels in the fifth row illustrate elbow displacement ( $\Delta$ elbow). Panels in the sixth row illustrate hand displacement ( $\Delta$ hand). For each row, the left panel represents the Bland and Altman plot and the right panel represents the linear regression plot. When assessed with the Kinect, PANU is excellently reliable but slightly overestimated, PAU is excellently reliable and very slightly underestimated, $\Delta$ trunk is excellently reliable and slightly underestimated, $\Delta$ shoulder is goodly reliable and slightly underestimated, $\Delta$ elbow is poorly reliable and slightly underestimated, $\Delta$ hand is moderately reliable and very slightly overestimated.

\section{List of abbreviations}

ICC: Intraclass Correlation Coefficient 
NVP: Number of Velocity Peaks

PAU: Proximal Arm Use

PANU: Proximal Arm NonUse

RMSE: Root-Mean-Square Error

\section{Declarations}

\section{Ethics approval and consent to participate}

This study was performed in accordance with the 1964 Declaration of Helsinki. The Institutional Review Board of EuroMov at the university of Montpellier approved the study (IRB-EM 1901C).

\section{Consent for publication}

Not applicable.

\section{Availability of data and materials}

The code and the datasets generated and analysed during the current study are available in the Open Science Framework repository, https://osf.io/ckrdp/.

\section{Competing interests}

The authors declare that they have no competing interests.

\section{Funding}

This study was supported by the LabEx NUMEV (ANR-10-LABX-0020) within the I-SITE MUSE.

\section{Authors' contributions}

G.F. designed the protocol, recorded and analysed the data, wrote the first version of the manuscript. D.M. and J.F. assisted G.F. in designing the protocol, analysing the data and provided guidance for improving the manuscript. All authors accepted the latest version of the manuscript.

\section{Acknowledgements}

We would like to thank Simon Pla for helping setting-up the experiment. We acknowledge Germain Faity for drawing the schema of the experimental conditions in Fig. 1.

\section{Bibliography}

1. Patterson TS, Bishop MD, McGuirk TE, Sethi A, Richards LG (2011) Reliability of Upper Extremity Kinematics While Performing Different Tasks in Individuals With Stroke. Journal of Motor Behavior 43:121-130

2. Alt Murphy M, Resteghini C, Feys P, Lamers I (2015) An overview of systematic reviews on upper extremity outcome measures after stroke. BMC Neurology 15:29

3. Bakhti KKA, Mottet D, Schweighofer N, Froger J, Laffont I (2017) Proximal arm non-use when reaching after a stroke. Neuroscience Letters 657:91-96

4. Roby-Brami A, Feydy A, Combeaud M, Biryukova EV, Bussel B, Levin MF (2003) Motor compensation and recovery for reaching in stroke patients. Acta Neurologica Scandinavica 107:369-381

5. Levin MF, Kleim JA, Wolf SL (2009) What Do Motor "Recovery" and "Compensation" Mean in Patients Following Stroke? Neurorehabil Neural Repair 23:313-319 
bioRxiv preprint doi: https://doi.org/10.1101/2022.01.18.476737; this version posted January 20,2022 . The copyright holder for this preprint (which was not certified by peer review) is the author/funder, who has granted bioRxiv a license to display the preprint in perpetuity. It is made available under aCC-BY-NC-ND 4.0 International license.

6. Subramanian SK, Yamanaka J, Chilingaryan G, Levin MF (2010) Validity of Movement Pattern Kinematics as Measures of Arm Motor Impairment Poststroke. Stroke 41:2303-2308

7. van Dokkum L, Hauret I, Mottet D, Froger J, Métrot J, Laffont I (2014) The Contribution of Kinematics in the Assessment of Upper Limb Motor Recovery Early After Stroke. Neurorehabil Neural Repair 28:4-12

8. Beebe Justin A., Lang Catherine E. (2009) Active Range of Motion Predicts Upper Extremity Function 3 Months After Stroke. Stroke 40:1772-1779

9. Chen H, Lin K, Liing R, Wu C, Chen C (2015) Kinematic measures of Arm-trunk movements during unilateral and bilateral reaching predict clinically important change in perceived arm use in daily activities after intensive stroke rehabilitation. Journal of NeuroEngineering and Rehabilitation 12:84

10. Cirstea MC, Levin MF (2000) Compensatory strategies for reaching in stroke. Brain 123:940-953

11. Levin MF, Liebermann DG, Parmet Y, Berman S (2016) Compensatory Versus Noncompensatory Shoulder Movements Used for Reaching in Stroke. Neurorehabil Neural Repair 30:635-646

12. Laffont I, Froger J, Jourdan C, et al (2020) Rehabilitation of the upper arm early after stroke: Video games versus conventional rehabilitation. A randomized controlled trial. Annals of Physical and Rehabilitation Medicine 63:173-180

13. Laver KE, Lange B, George S, Deutsch JE, Saposnik G, Crotty M (2017) Virtual reality for stroke rehabilitation. Cochrane Database of Systematic Reviews.

https://doi.org/10.1002/14651858.CD008349.pub4

14. Springer S, Seligmann GY (2016) Validity of the Kinect for Gait Assessment: A Focused Review. Sensors 16:194

15. Corti A, Giancola S, Mainetti G, Sala R (2016) A metrological characterization of the Kinect V2 time-of-flight camera. Robotics and Autonomous Systems 75:584-594

16. Mortazavi F, Nadian-Ghomsheh A (2018) Stability of Kinect for range of motion analysis in static stretching exercises. PLOS ONE 13:e0200992

17. Çubukçu B, Yüzgeç U, Zileli R, Zileli A (2020) Reliability and validity analyzes of Kinect V2 based measurement system for shoulder motions. Medical Engineering \& Physics 76:20-31

18. Kuster RP, Heinlein B, Bauer CM, Graf ES (2016) Accuracy of KinectOne to quantify kinematics of the upper body. Gait \& Posture 47:80-85

19. Scano A, Mira RM, Cerveri P, Molinari Tosatti L, Sacco M (2020) Analysis of Upper-Limb and Trunk Kinematic Variability: Accuracy and Reliability of an RGB-D Sensor. MTI 4:14

20. Zulkarnain RF, Kim G-Y, Adikrishna A, Hong HP, Kim YJ, Jeon I-H (2017) Digital data acquisition of shoulder range of motion and arm motion smoothness using Kinect v2. Journal of Shoulder and Elbow Surgery 26:895-901

21. Cai L, Ma Y, Xiong S, Zhang Y (2019) Validity and Reliability of Upper Limb Functional Assessment Using the Microsoft Kinect V2 Sensor. Applied Bionics and Biomechanics 2019:e7175240 
22. Mentiplay BF, Perraton LG, Bower KJ, Pua Y-H, McGaw R, Heywood S, Clark RA (2015) Gait assessment using the Microsoft Xbox One Kinect: Concurrent validity and inter-day reliability of spatiotemporal and kinematic variables. Journal of Biomechanics 48:2166-2170

23. Humadi A, Nazarahari M, Ahmad R, Rouhani H (2021) In-field instrumented ergonomic risk assessment: Inertial measurement units versus Kinect V2. International Journal of Industrial Ergonomics 84:103147

24. Bakhti KKA, Laffont I, Muthalib M, Froger J, Mottet D (2018) Kinect-based assessment of proximal arm non-use after a stroke. J NeuroEngineering Rehabil 15:104

25. Scano A, Molteni F, Molinari Tosatti L (2019) Low-Cost Tracking Systems Allow Fine Biomechanical Evaluation of Upper-Limb Daily-Life Gestures in Healthy People and Post-Stroke Patients. Sensors 19:1224

26. Sheng B, Wang $X$, Hou M, Huang J, Xiong S, Zhang Y (2020) An automated system for motor function assessment in stroke patients using motion sensing technology: A pilot study. Measurement 161:107896

27. Sheng B, Wang X, Xiong S, Hou M, Zhang Y (2019) Kinematic Metrics for Upper-limb Functional Assessment of Stroke Patients. In: 2019 International Conference on Intelligent Informatics and Biomedical Sciences (ICIIBMS). pp 45-51

28. Faity G, Mottet D, Pla S, Froger J (2021) The reserve of joint torque determines movement coordination. Sci Rep 11:23008

29. Nichols JK, Sena MP, Hu JL, O’Reilly OM, Feeley BT, Lotz JC (2017) A Kinect-based movement assessment system: marker position comparison to Vicon. Computer Methods in Biomechanics and Biomedical Engineering 20:1289-1298

30. Merriaux P, Dupuis Y, Boutteau R, Vasseur P, Savatier X (2017) A Study of Vicon System Positioning Performance. Sensors 17:1591

31. Da Gama A, Fallavollita P, Teichrieb V, Navab N (2015) Motor Rehabilitation Using Kinect: A Systematic Review. Games for Health Journal 4:123-135

32. Lupinacci G, Gatti G, Angilica A, Muzzupappa M (2016) Kinect V2 for Upper Limb Rehabilitation Applications - A Preliminary Analysis on Performance Evaluation: In: Proceedings of the 9th International Joint Conference on Biomedical Engineering Systems and Technologies. SCITEPRESS - Science and and Technology Publications, Rome, Italy, pp 131-138

33. Xu X, Robertson M, Chen KB, Lin J, McGorry RW (2017) Using the Microsoft Kinect ${ }^{\mathrm{TM}}$ to assess 3D shoulder kinematics during computer use. Applied Ergonomics 6

34. Arun KS, Huang TS, Blostein SD (1987) Least-Squares Fitting of Two 3-D Point Sets. IEEE Transactions on Pattern Analysis and Machine Intelligence PAMI-9:698-700

35. Nghia Ho (2021), GitHub repository, https://github.com/nghiaho12/rigid_transform_3D.

36. Altman DG, Bland JM (1983) Measurement in Medicine: The Analysis of Method Comparison Studies. Journal of the Royal Statistical Society: Series D (The Statistician) 32:307-317 
bioRxiv preprint doi: https://doi.org/10.1101/2022.01 18.476737; this version posted January 20, 2022. The copyright holder for this preprint (which was not certified by peer review) is the author/funder, who has granted bioRxiv a license to display the preprint in perpetuity. It is made available under aCC-BY-NC-ND 4.0 International license.

37. Koo TK, Li MY (2016) A Guideline of Selecting and Reporting Intraclass Correlation Coefficients for Reliability Research. J Chiropr Med 15:155-163

38. Schwarz Anne, Kanzler Christoph M., Lambercy Olivier, Luft Andreas R., Veerbeek Janne M. (2019) Systematic Review on Kinematic Assessments of Upper Limb Movements After Stroke. Stroke 50:718-727

39. Cutti AG, Paolini G, Troncossi M, Cappello A, Davalli A (2005) Soft tissue artefact assessment in humeral axial rotation. Gait \& Posture 21:341-349

40. Xu X, McGorry RW (2015) The validity of the first and second generation Microsoft Kinect ${ }^{\mathrm{TM}}$ for identifying joint center locations during static postures. Applied Ergonomics 49:47-54

41. Cai L, Liu D, Ma Y (2021) Placement Recommendations for Single Kinect-Based Motion Capture System in Unilateral Dynamic Motion Analysis. Healthcare 9:1076

42. Collins KC, Kennedy NC, Clark A, Pomeroy VM (2018) Kinematic Components of the Reach-toTarget Movement After Stroke for Focused Rehabilitation Interventions: Systematic Review and Meta-Analysis. Front Neurol. https://doi.org/10.3389/fneur.2018.00472

43. Levin MF (1996) Interjoint coordination during pointing movements is disrupted in spastic hemiparesis. Brain 119:281-293

44. Ma Y, Liu D, Cai L (2020) Deep Learning-Based Upper Limb Functional Assessment Using a Single Kinect v2 Sensor. Sensors 20:1903

45. Jun C, Lee JY, Kim BH, Noh SD (2019) Automatized modeling of a human engineering simulation using Kinect. Robotics and Computer-Integrated Manufacturing 55:259-264

46. Moon S, Park Y, Ko DW, Suh IH (2016) Multiple Kinect Sensor Fusion for Human Skeleton Tracking Using Kalman Filtering. International Journal of Advanced Robotic Systems 13:65

47. Ryselis K, Petkus T, Blažauskas T, Maskeliūnas R, Damaševičius R (2020) Multiple Kinect based system to monitor and analyze key performance indicators of physical training. Hum Cent Comput Inf Sci 10:51

48. Beshara P, Chen JF, Read AC, Lagadec P, Wang T, Walsh WR (2020) The Reliability and Validity of Wearable Inertial Sensors Coupled with the Microsoft Kinect to Measure Shoulder Range-ofMotion. Sensors 20:7238

49. Chen P, Du Y, Shih C, Yang L, Lin H, Fan S (2016) Development of an upper limb rehabilitation system using inertial movement units and kinect device. In: 2016 International Conference on Advanced Materials for Science and Engineering (ICAMSE). pp 275-278

50. Jatesiktat P, Anopas D, Ang WT (2018) Personalized Markerless Upper-Body Tracking with a Depth Camera and Wrist-Worn Inertial Measurement Units. In: 2018 40th Annual International Conference of the IEEE Engineering in Medicine and Biology Society (EMBC). pp 1-6

51. Li R, Si W, Weinmann M, Klein R (2019) Constraint-Based Optimized Human Skeleton Extraction from Single-Depth Camera. Sensors (Basel) 19:E2604

52. Mottet D, van Dokkum LEH, Froger J, Gouaich A, Laffont I (2017) Trajectory formation principles are the same after mild or moderate stroke. PLoS ONE 12:e0173674 
53. Murphy MA, Willén C, Sunnerhagen KS (2011) Kinematic Variables Quantifying Upper-Extremity Performance After Stroke During Reaching and Drinking From a Glass. Neurorehabil Neural Repair 25:71-80

54. Jones T. A. (2017) Motor compensation and its effects on neural reorganization after stroke. In: Nature Reviews Neuroscience.

https://www.researchgate.net/publication/315526603_Motor_compensation_and_its_effects _on_neural_reorganization_after_stroke. Accessed 30 Oct 2018

55. Popović MD, Kostić MD, Rodić SZ, Konstantinović LM (2014) Feedback-Mediated Upper Extremities Exercise: Increasing Patient Motivation in Poststroke Rehabilitation. BioMed Research International 2014:e520374

56. Cirstea C.M., Ptito A., Levin M.F. (2006) Feedback and Cognition in Arm Motor Skill Reacquisition After Stroke. Stroke 37:1237-1242

57. Subramanian SK, Massie CL, Malcolm MP, Levin MF (2010) Does Provision of Extrinsic Feedback Result in Improved Motor Learning in the Upper Limb Poststroke? A Systematic Review of the Evidence. Neurorehabil Neural Repair 24:113-124

58. Rodrigues MRM, Slimovitch M, Chilingaryan G, Levin MF (2017) Does the Finger-to-Nose Test measure upper limb coordination in chronic stroke? Journal of NeuroEngineering and Rehabilitation 14:6

59. Subramanian SK, Cross MK, Hirschhauser CS (2020) Virtual reality interventions to enhance upper limb motor improvement after a stroke: commonly used types of platform and outcomes. Disability and Rehabilitation: Assistive Technology 1-9

60. Desmarais $Y$, Mottet $D$, Slangen $P$, Montesinos $P$ (2021) A review of $3 D$ human pose estimation algorithms for markerless motion capture. Computer Vision and Image Understanding 212:103275

61. Cao Z, Hidalgo G, Simon T, Wei S-E, Sheikh Y (2021) OpenPose: Realtime Multi-Person 2D Pose Estimation Using Part Affinity Fields. IEEE Transactions on Pattern Analysis and Machine Intelligence 43:172-186

62. Pavllo D, Feichtenhofer C, Grangier D, Auli M (2019) 3D Human Pose Estimation in Video With Temporal Convolutions and Semi-Supervised Training. In: 2019 IEEE/CVF Conference on Computer Vision and Pattern Recognition (CVPR). pp 7745-7754

63. Iskakov K, Burkov E, Lempitsky V, Malkov Y (2019) Learnable Triangulation of Human Pose. In: 2019 IEEE/CVF International Conference on Computer Vision (ICCV). pp 7717-7726

64. Tölgyessy M, Dekan M, Chovanec L', Hubinský P (2021) Evaluation of the Azure Kinect and Its Comparison to Kinect V1 and Kinect V2. Sensors 21:413 